$>$ L'immunosurveillance des muqueuses et de la peau est assurée par des cellules dendritiques spécialisées dans la capture, le transport et la présentation des antigènes aux lymphocytes $T$ des ganglions lymphatiques drainants. Les cellules dendritiques de ces interfaces épithéliales, les cellules de Langerhans, ont été longtemps reconnues comme prototypes de cellules dendritiques immunostimulatrices, spécialisées dans l'activation de lymphocytes T en réponse à l'immunisation avec des antigènes vaccinaux ou à l'infection par des pathogènes. Ce concept est remis en question par des données récentes montrant que les cellules de Langerhans résidant dans ces épithélium ne sont pas indispensables à l'induction d'une immunité T spécifique et pourraient participer au maintien de la tolérance périphérique. $\varepsilon n$ revanche, les cellules dendritiques recrutées au site d'immunisation par certains adjuvants et pathogènes délivrés par voie muqueuse ou cutanée permettent l'induction de lymphocytes T CD $8^{+}$ cytotoxiques d'intérêt vaccinal. <

\section{Cellules dendritiques de la peau et des muqueuses}

\section{Diversité des cellules dendritiques épithéliales}

La peau et les muqueuses sont à l'interface avec le milieu extérieur et assurent le maintien de l'intégrité tissulaire face aux effets pathogènes d'agents biologiques, chimiques ou physiques de l'environnement. La peau et les muqueuses à épithélium pluristratifié (muqueuses buccale, vaginale, rectale) comportent plusieurs populations de cellules dendritiques (DC), localisées dans les couches épithéliales et dans le tissu conjonctif sous-jacent (Figure 1). Les DC des couches basale et supra-basale de l'épithélium, c'est-à-dire les cellules de Langerhans $(C L)$, forment un réseau par leurs dendrites en contact avec les cellules épithéliales. Les $\mathrm{CL}$ constituent, à l'état basal, les seules cellules présentatrices de l'antigène (CPA) de l'épithélium [1].

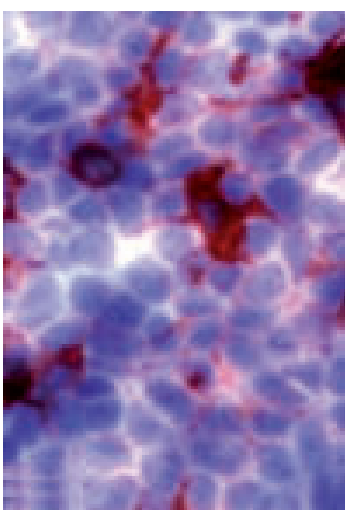

M. Le Borgne, B. Dubois : Inserm U851, Immunité, Infection et Vaccination, IFR128, 21, avenue Tony Garnier, 69365 Lyon Cedex 07, France. Université Claude Bernard Lyon 1, IFR128 BioSciences Lyon-Gerland, 69365 Lyon, France.

D. Kaiserlian : Inserm U851, Immunité, Infection et Vaccination, IFR128, 21, avenue Tony Garnier,

D'autres types de DC, dénommées DC dermiques ou interstitielles, moins bien caractérisées, sont présentes dans le tissu conjonctif (le derme cutané et la lamina propria muqueuse) [2]. Cer69365 Lyon Cedex 07, France. Université Claude Bernard Lyon 1, IFR128 BioSciences Lyon-Gerland, 69365 Lyon, France.

Hospices Civils de Lyon, Hôpital Edouard Herriot, 69003 Lyon, France.

kaiserlian@cervi-lyon.inserm.fr taines de ces cellules sont phénotypiquement et fonctionnellement similaires aux monocytes et pourraient constituer des précurseurs de CL [3]. La distribution des $D C$ dans les tissus muqueux varie selon la nature de l'épithélium de revêtement et l'état inflammatoire du tissu. Les DC des muqueuses à épithélium monostratifié (intestin) ou pseudostratifié (poumons) sont localisées surtout dans la lamina propria [4], et sont peu représentées dans l'épithélium, bien que l'émission de dendrites entre une monocouche de cellules épithéliales absorbantes leur permette de capturer des antigènes $(\mathrm{Ag})$ de la lumière [5]. Les $D C$ plasmacytoïdes $(p D C)$ sont des $D C$ non conventionnelles caractérisées par leur morphologie (similaire à celle des plasmocytes) et leur capacité à produire des quantités importantes d'interféron de type I en réponse aux virus. 
Les pDC sont présentes dans le sang et les organes lymphoïdes mais ont aussi été identifiées dans les muqueuses (intestinale et bronchique) et dans le derme au cours de pathologies inflammatoires [6].

\section{Dynamique des cellules dendritiques épithéliales}

Les DC résidant dans les interfaces épithéliales sont dans un état dit «immature», caractérisé par une forte capacité de capture et d'internalisation d'antigènes [6]. Des signaux de danger, comme la production de cytokines et de chimiokines pro-inflammatoires,

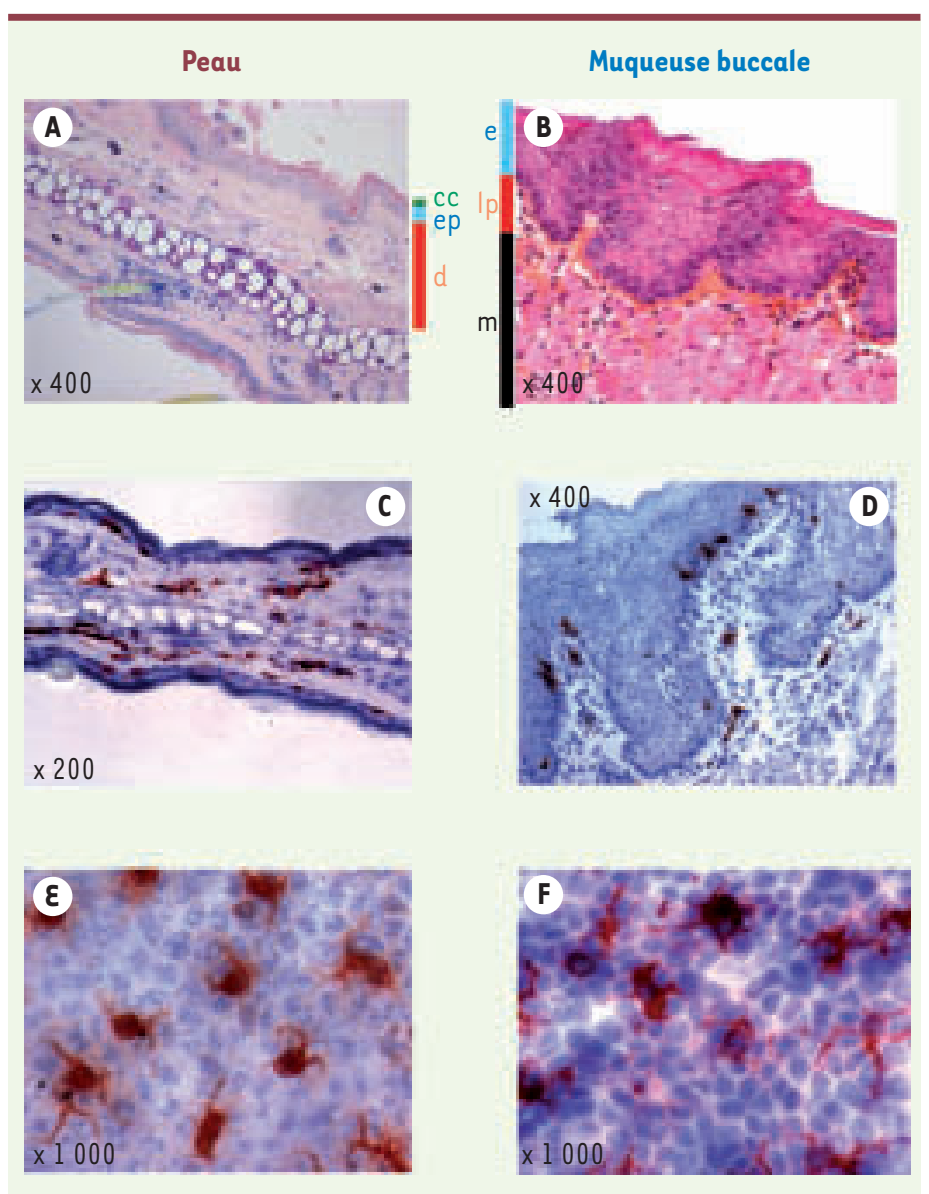

Figure 1. Distribution des cellules dendritiques de la peau et de la muqueuse buccale. Structure histologique de la peau (oreille) (A) et de la muqueuse buccale $(B)$ de souris. Les coupes transversales de peau marquées à l'hématoxyline éosine $(A, C)$ révèlent que l'épiderme (ep) est mince et recouvert d'une couche cornée (cc), alors que l'épithélium buccal est épais et pauvre en couche cornée. Le tissu conjonctif sous-jacent à l'épithélium de la muqueuse (lamina propria, Ip) ou de la peau (derme, d), est riche en vaisseaux sanguins et en cellules mononuclées et recouvre une couche musculaire $(\mathrm{m})$. C-F. Les DC de l'oreille ( $\boldsymbol{C}$, $\varepsilon$ ) et de la muqueuse buccale ( $D, F)$ exprimant des molécules du CMH de classe II sont localisées dans le derme superficiel et dans l'épithélium en position suprabasale $(C-D)$ où elles forment un réseau $(\boldsymbol{E}-\boldsymbol{F})$. Les $D C$ de l'épithélium (cellules de Langerhans) sont caractérisées par l'expression de marqueurs spécifiques comme l' $\varepsilon$-cadhérine et la Langérine, et présentent dans leur cytoplasme des organites multilamellaires, les granules de Birbeck [6]. peuvent induire la maturation fonctionnelle des DC, ce qui se traduit par leur migration vers les ganglions lymphatiques drainants, où elles acquièrent la capacité de présenter l'antigène et d'activer les lymphocytes $T$ naïs. Ces signaux de maturation peuvent provenir de virus, de bactéries ou de certains de leur composants, qui agissent soit directement en se liant à des récepteurs portés par les DC comme les TLR (toll-like receptor), soit indirectement via la production, dans le microenvironnement, de cytokines pro-inflammatoires (TNF- $\alpha, I L-1 \beta$ ). Le fait que les DC interstitielles et les $\mathrm{CL}$ émigrant des tissus colonisent des zones $T$ distinctes des ganglions [7], suggère qu'elles pourraient exercer des rôles complémentaires, bien que l'impact de cette compartimentation sur la réponse immunitaire reste à clarifier. Ainsi, les ganglions drainant la peau et les muqueuses comportent à l'état basal des DC dérivant des $D C$ interstitielles et une population de DC dérivant des CL. Trois autres populations de DC, arrivant par voie sanguine, ont été identifiées dans les organes lymphoïdes secondaires: des $D C C^{2} 11 b^{+} C D 8 \alpha^{-}$, des $D C$ CDIlb-CD $8 \alpha^{+}$, et des pDC [8]. Leurs rôles respectifs dans l'induction de la réponse immunitaire sont mal définis à ce jour.

Renouvellement des cellules dendritiques épithéliales Les DC sont des cellules d'origine hématopoïétique. On peut induire in vitro la différenciation, en $D C$ de type interstitiel et en $\mathrm{CL}$, de précurseurs $\mathrm{CD} 34^{+}$issus de la moelle osseuse et du sang de cordon, ou de monocytes circulants. In vivo, il a été montré que le passage de monocytes à travers l'endothélium vasculaire dans la peau peut s'effectuer sous l'influence de stimulus proinflammatoires produits par l'injection intradermique d'Ag particulaires, et aboutit à leur différenciation en $D C$ capables, après capture de l'Ag, de migrer dans les ganglions lymphatiques drainants [9]. Le renouvellement des DC des épithélium s'effectue à partir de précurseurs dont l'origine dépend de la nature et de l'intensité des signaux de stress et/ou de danger auxquels le tissus est soumis, et donc des chimiokines et des cytokines pro-inflammatoires produites localement. Ainsi, il a été observé chez la souris et chez l'homme que les CL de l'hôte soumis à une irradiation puis recevant une greffe de moelle osseuse, persistent pendant au moins 18 mois, contrairement aux autres populations de DC [10], ce qui montre que les CL peuvent être renouvelées dans l'épiderme, en proliférant ou en se différenciant à partir de précurseurs locaux radio-résistants. En revanche, si la peau est le siège d'une inflammation, les $\mathrm{CL}$ sont renouvelées à partir de précurseurs myéloïdes circulants [10], les monocytes $\mathrm{Grl}^{+}[11]$. 


\section{Cellules dendritiques et induction de la réponse immunitaire T CD8 ${ }^{+}$}

\section{Les $D C$ matures activées sont nécessaires}

à l'induction de LT CD8 ${ }^{+}$cytotoxiques

Le rôle clé joué par les $D C$ dans l'initiation de réponses immunitaires $T$ $\mathrm{CD}^{+}{ }^{+}$et $\mathrm{CD} 88^{+}$spécifiques d'antigène a été proposé par Ralph Steinman dans les années 1970. Par la suite, de nombreuses études ont montré que les $D C$ sont capables de présenter, outre des antigènes endogènes, des antigènes exogènes solubles sur les molécules du CMH (complexe majeur d'histocompatibilité) de classe I (présentation croisée) et d'induire la différenciation de lymphocytes $T$ (LT) CD8 $8^{+}$spécifiques et cytotoxiques (CTL). Ainsi, les DC, différenciées in vitro à partir de précurseurs issus de la moelle osseuse, ou isolées de la peau [12], puis chargées avec un antigène, induisent des $C T L C D 8^{+}$spécifiques in vivo, après leur transfert chez des souris naïves. En outre, la déplétion in vivo des $D C$, qu'entraîne l'injection de toxine diphtérique à des souris transgéniques exprimant le récepteur de cette toxine sous le contrôle du promoteur de la molécule CDllc (un marqueur de DC), empêche I'induction de réponses CTL chez des souris soumises à une infection virale ou parasitaire [13].

Si les DC à l'état basal (immatures) peuvent apprêter des antigènes sur la voie du CMH de classe I, seuls certains signaux d'activation induisent leur maturation fonctionnelle et leur permettent d'induire la différenciation des $L T C D 8^{+}$naïfs en CTL. Les DC peuvent être activées soit directement, par exemple lors d'infections, ou via l'engagement de récepteurs de l'immunité innée (TLR, récepteurs scavenger ${ }^{1}$ ), soit indirectement, via la production de cytokines pro-inflammatoires (IL-1 $\beta$, IL-18). Cependant, la différenciation des $L T C D 4^{+}$en $L T$ effecteurs nécessite l'activation directe des DC via l'engagement de leur TLR par des constituants d'agents pathogènes. De la même façon, l'induction d'une réponse CD8 $8^{+}$CTL par les DC nécessite leur activation par des signaux dérivés des pathogènes ou des LT auxiliaires [14].

Les cellules dendritiques résidant dans les épithélium ont un rôle limité dans l'induction des réponses CTL

Depuis la découverte des $D C$, de nombreuses études réalisées in vitro et in vivo, chez la souris et chez l'homme, ont abouti au concept que les CL sont immunostimulatrices et spécialisées dans l'activation des LT naïfs. Ce dogme a récemment été remis en question, grâce au développement d'anticorps capables de cibler les $D C$, ou de souris transgéniques permettant de suivre les $\mathrm{CL}$ ou d'induire leur ablation conditionnelle in vivo. Ainsi, dans un modèle d'infection cutanée avec le virus de l'herpès, il apparaît que le rôle des CL de l'épiderme se limite au transport des Ag viraux vers les ganglions lymphatiques, et que ce sont les $D C$ ganglionnaires qui sont responsa-

${ }^{1}$ Scavenger dont la traduction littérale est éboueur, désigne des récepteurs des macrophages, par exemple CD36, ou SR-B1, ou le récepteur du mannose.

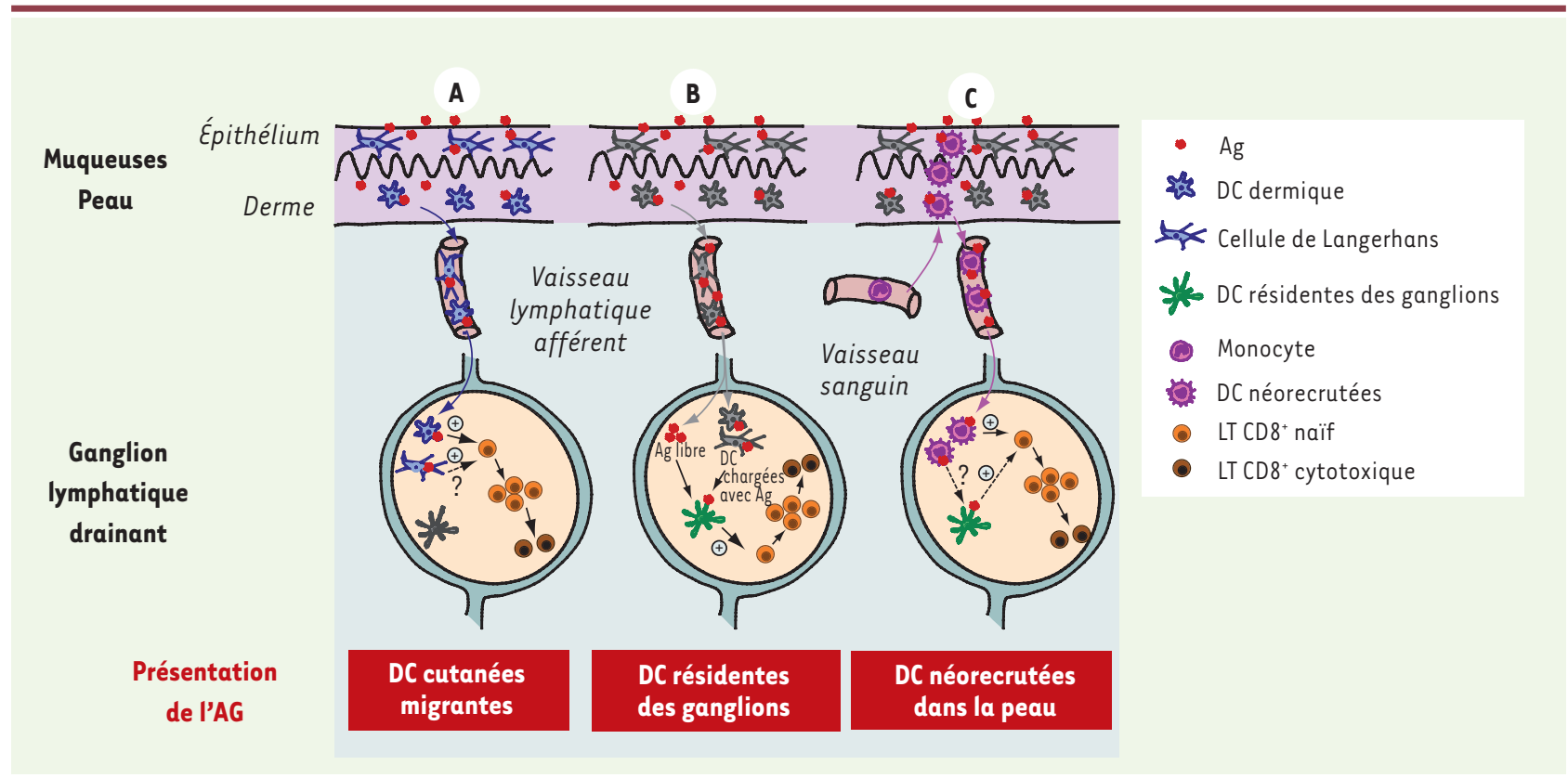

Figure 2. Nature des DC responsables de la présentation d'Ag administrés par voie muqueuse ou cutanée. Les Ag administrés via les muqueuses ou la peau, peuvent être capturés et présentés aux LT CD $8^{+}$par des DC des interfaces épithéliales, migrant dans les ganglions lymphatiques ( $A$ ). L’Ag transporté via la lymphe jusqu'aux ganglions drainants sous forme soluble ou par les DC des tissus épithéliaux, peut être présenté aux LT CD8 ${ }^{+}$par les $D C C D 8 \alpha^{+}$résidant dans les ganglions drainants (B). L'Ag peut induire le recrutement de DC capables de le transporter depuis le site d'immunisation jusqu'aux ganglions drainants et soit de présenter directement l'Ag aux LT CD8 $8^{+}$ou de le transférer à des DC des ganglions (C). 
bles de la présentation antigénique aux $\operatorname{LT}_{\operatorname{CD} 8}{ }^{+}[15,16]$. Par ailleurs, la déplétion des $\mathrm{CL}$ au moment d'une sensibilisation cutanée avec un haptène n'empêche pas le développement de la réaction d'hypersensibilité retardée de contact, et peut même provoquer une augmentation de la réponse inflammatoire $[7,17,18]$. Ces données permettent aujourd'hui de penser que les CL (de la peau et de certaines muqueuses): (1) ne sont pas nécessairement impliquées dans l'induction de réponses $T$ effectrices; (2) que le cas échéant, leur fonction pourrait se limiter au simple transport de l'Ag vers les ganglions drainants; et (3) qu'elles sont susceptibles d'exercer un contrôle négatif des réponses T proinflammatoires (par exemple via la transduction de signaux inhibiteurs et/ou l'activation de cellules régulatrices/suppressives).

En revanche, un faisceau d'arguments montre que des DC interstitielles des muqueuses (nasale et vaginale) sont capables de présenter l'Ag ex vivo à des

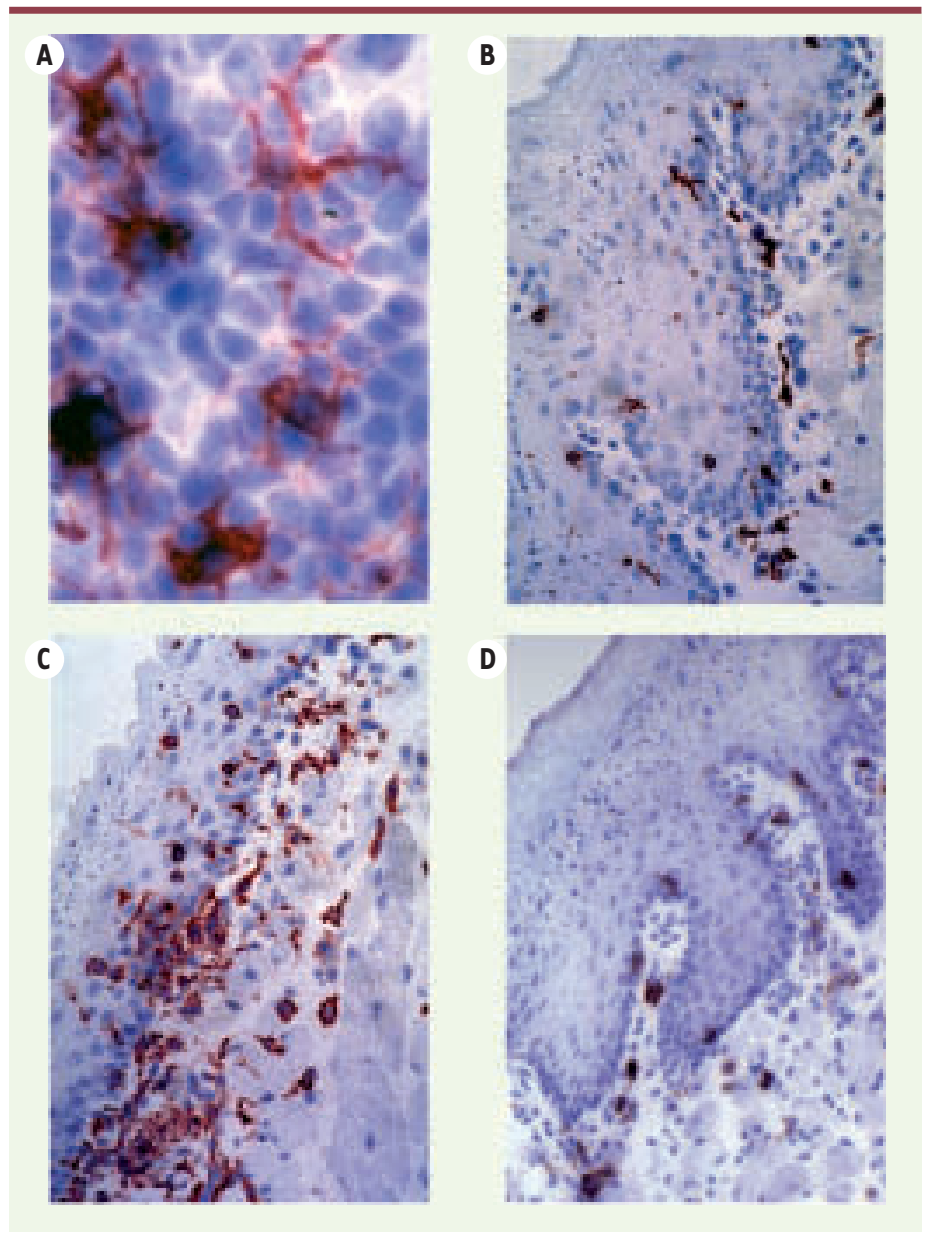

Figure 3. Recrutement de $D C$ dans les muqueuses. Réseau de $C L$ exprimant des molécules du CMH de classe II dans un feuillet d'épithélium buccal de souris ( $A$ ). Les coupes transversales de muqueuse buccale à l'état basal montrent la localisation des CL dans les couches suprabasales de l'épithélium et des DC interstitielles à la jonction dermo-epithéliale (B). Deux heures après injection intradermique de la nucléoprotéine du virus de la rougeole $(C-D)$, on note un recrutement massif de $C L$ et de $D C$ dans la muqueuse buccale de souris normales (C), qui n'est pas observé chez des souris déficientes en CCR6 (D). Grossissement final : x 400.
LT CD8 ${ }^{+}$spécifiques, et d'induire une immunité T spécifique d'antigènes infectieux $[19,20]$ (Figure 2A). Les données obtenues dans des modèles d'infection ont montré que les $D C C D 8 \alpha^{+}$résidentes des ganglions sont efficaces pour présenter l'Ag et induire l'activation de $\operatorname{LTCD} 8^{+}$cytotoxiques $[15,19]$. Cela suggère que des $D C$ matures résidentes des ganglions pourraient capturer des Ag solubles ou particulaires ayant diffusé librement jusqu'aux ganglions drainants [21], ou ayant été transportés par des DC depuis les tissus épithéliaux jusqu’aux ganglions, via la lymphe (Figure 2B).

\section{Cellules dendritiques néorecrutées et induction de réponses $\mathrm{T}_{\mathrm{CD}} 8^{+}$in vivo}

Plusieurs groupes ont observé que l'exposition des muqueuses et/ou de la peau à des virus, des bactéries [22], des adjuvants tels que la toxine cholérique et la flagelline [23, 24], à la nucléoprotéine du virus de la rougeole (NP) $[25,26]$, ou à des produits chimiques à pouvoir sensibilisant $[26,27]$, provoquait une accumulation de $D C$ au site d'immunisation. Le fait que la plupart de ces Ag soient de puissants immunogènes capables d'activer des CTL CD $8^{+}$spécifiques in vivo en l'absence de LT CD4 $4^{+}$a permis de postuler que leur immunogénicité pouvait être dépendante de $D C$ néorecrutées.

Ainsi, nous avons montré chez la souris, que l'application topique de l'haptène 2,4-dinitrofluorobenzène (DNFB) sur la peau ou la muqueuse buccale, ou l'injection intradermique de la NP du virus de la rougeole, provoquait une augmentation de la sécrétion locale de la chimiokine CCL20. Ce type d'immunisation conduit à un recrutement rapide et transitoire de $D C$, via un mécanisme dépendant du récepteur CCR6 (récepteur de CCL20 exprimé par les DC immatures mais pas par les DC matures) (Figure 3) [26]. Cette accumulation de $D C$ est précédée d'un recrutement à partir du sang de monocytes $\mathrm{Grl}^{+}$, le précurseur majoritaire des $D C$ en conditions inflammatoires [11]. Nous avons démontré que l'haptène DNFB et la NP possèdent des propriétés adjuvantes permettant l'induction de CTL CD $8^{+}$contre une protéine (ovalbumine) co-administrée par voie intradermique, via un mécanisme dépendant de la voie CCR6/CCL20. Cette observation a été confirmée pour d'autres adjuvants (flagelline, toxine cholérique, poly [I: C]) administrés via la muqueuse buccale ou la peau. Des expériences de transfert adoptif et de déplétion in vivo des monocytes ou utilisant des souris chimères et des souris génétiquement modifiées, ont permis de démontrer formellement que les $D C$ néorecrutées par ces adjuvants dans les muqueuses et la peau, dérivent de monocytes circulants $\mathrm{Gr}^{+}$et sont responsables de la 
présentation directe de l'Ag aux LT CD8 ${ }^{+}$naïfs. Les DC néorecrutées par les adjuvants dans les couches épithéliales et sub-épithéliales de la peau et des muqueuses, peuvent donc se différencier rapidement in situ à partir des monocytes sanguins et pourraient représenter les DC immunostimulatrices responsables de l'établissement d'une réponse CTL efficace [26]. L'interaction CCR6/CCL20 peut contribuer également à la rétention de $D C$ dans l'épithélium du dôme des plaques de Peyer après infection orale avec Salmonella typhimirium, et conduire à l'activation de LT CD4 ${ }^{+}$spécifiques [28]. Enfin, après une infection cutanée par Leishmania major, des monocytes rapidement recrutés dans le derme et les ganglions drainants sont capables de se différencier en DC [29]. Ces DC néorecrutées constituent les seules $D C$ infectées dans les ganglions et sont capables de présenter les antigènes parasitaires aux LT.

Ainsi, qu'il s'agisse de l'induction de réponses T CD $4^{+}$ou $C D 8^{+}$effectrices, il apparaît que ce sont les $D C$ recrutées dans les muqueuses et la peau sous l'influence de stimulus pro-inflammatoires, et non les DC présentes à l'état basal, qui jouent un rôle essentiel dans l'induction de réponses T spécifiques vis-à-vis d'antigènes administrés par voie muqueuse ou cutanée (Figure 2C et Figure 4). Ce nouveau concept revêt toute son importance pour l'induction de la réponse immunitaire consécutive à une infection ou une vaccination. Pourquoi les $D C$ néorecrutées et non les résidentes sont-elles immunostimulatrices? L'une des hypothèses est que ces $D C$ néorecrutées n'ont pas subi l'influence du microenvironnement épithélial riche en cytokines à fonction suppressive (IL-10, TGF- $\beta$ ) et sont à un stade de différenciation plus précoce, qui les rend plus sensibles aux signaux pro-inflammatoires délivrés par l'adjuvant ou l'agent pathogène.

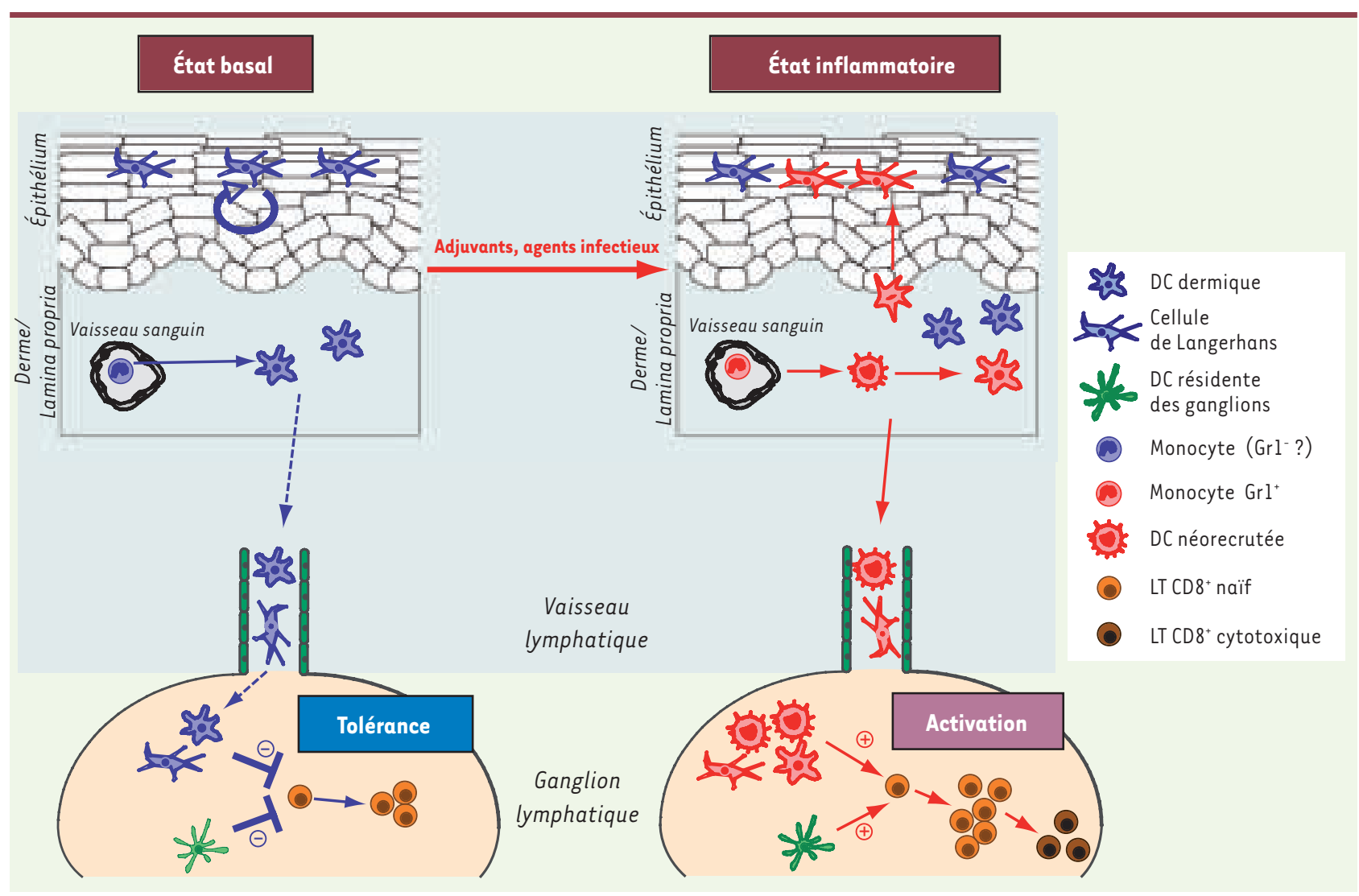

Figure 4. Propriétés fonctionnelles des DC épithéliales. A. À l'homéostasie, les CL de l'épiderme se renouvellent à partir de précurseurs locaux radiorésistants [10]. Les $D C$ dermiques se différencient à partir de précurseurs circulants, qui pourraient être les monocytes Gr ${ }^{-}$recrutés dans les tissus à l'état basal. Leur recrutement serait induit par le couple chimiokine récepteur $C X_{3} C L 1 / C X_{3} C R 1$ [35]. Les $D C$ résidentes des épithéliums migrent continuellement vers les ganglions drainants pour y présenter des $\mathrm{Ag}$ du soi ou de l'environnement, et pourraient contribuer à l'induction et/ou au maintien de la tolérance périphérique. B. En situation inflammatoire (infection, vaccination), des monocytes Grl ${ }^{+}$circulants migrent dans le derme $[11,26,35]$. Ils peuvent alors se différencier dans le derme en macrophages tissulaires, ou en DC capables soit de migrer rapidement dans les ganglions drainants [9], soit de se différencier en CL avant ou après avoir migré dans l'épiderme [11]. Les récepteurs CCR2 et CCR6 et leurs ligands respectifs (CCL2/CCL13 et CCL20), induiraient la migration séquentielle des précurseurs de DC du sang dans le derme, puis du derme dans l'épiderme $[10,26,36]$. Les DC néorecrutées sont responsables de l'activation de LT CD8+ spécifiques et de leur différenciation en LT cytotoxiques dans les ganglions lymphatiques drainants. Les DC résidentes des ganglions lymphatiques pourraient également présenter l'Ag aux LT CD8+. 


\section{Cellules dendritiques des épithélium et tolérance périphérique}

Si les $D C$ qui résident dans les tissus épithéliaux ne sont pas essentielles à l'induction de réponses CTL, elles semblent cependant intervenir dans le maintien de la tolérance périphérique. En effet, la présentation de l'Ag par des DC à l'état basal, en l'absence de signal d'activation, conduit à une tolérance. Ainsi, il a été montré dans un modèle de souris transgéniques, qu'un Ag exprimé à l'état basal dans le pancréas est capturé et présenté par des DC CD8 $\alpha^{+}$des ganglions drainants, entraînant la déplétion des $L T C D 8^{+}$spécifiques [30]. De façon comparable, un $\mathrm{Ag}$ conjugué à l'anticorps anti-DEC205 (récepteur exprimé à la surface des $D C C D 8 \alpha^{+}$, des $D C$ interstitielles et des $C L$ ) est efficacement capturé et présenté par les $D C$ des ganglions lymphatiques, ce qui conduit à une délétion des LT spécifiques [31]. De même, la transduction d'Ag viraux par les $D C$ peut conduire à l'induction d'une tolérance spécifique des LT CD8+ [14].

Les DC immatures des épithélium pourraient donc à l'état basal participer au maintien de la tolérance périphérique (Figure 4). Ainsi, les $D C$ des muqueuses bronchique et intestinale présentent des propriétés tolérogènes (production d'IL-10 et de TGF- $\beta$, induction de LT régulateurs) et pourraient participer au maintien de la tolérance périphérique aux aéroallergènes [32], aux Ag alimentaires et aux bactéries de la flore commensale [33]. Les CL pourraient également exercer des fonctions tolérogènes, puisque leur déplétion in vivo au moment de l'immunisation topique par un haptène peut conduire à une augmentation de la réponse d'hypersensibilité retardée de contact [18]. Par ailleurs, il est proposé que les $C L$ de la muqueuse buccale participent à la tolérance systémique à des allergènes protéiques, lors des protocoles de désensibilisation par voie sublinguale [34]. Les propriétés tolérogènes des $D C$ résidant dans les tissus épithéliaux résultent probablement de leur exposition prolongée au microenvironnement riche en cytokines comme l'IL-10 ou le TGF- $\beta$.

\section{Conclusions et perspectives}

L'identification des sous-populations de DC de la peau et des muqueuses à cibler pour induire l'activation et la différenciation de cellules T CD8 $8^{+}$CTL représente un enjeu majeur pour la mise en œuvre de nouveaux vaccins anti-infectieux ou anti-tumoraux. Ces avancées récentes sur la dynamique des $D C$ néorecrutées et leur fonction immunostimulatrice devraient permettre de cribler les adjuvants capables de promouvoir la sécrétion de CCL20 et de sélectionner ceux capables de provoquer le recrutement de monocytes et leur différenciation en DC immunostimulatrices. $\diamond$

\section{SUMMARY}

Dendritic cells of mucosa and skin: "recruited for vaccination"

Mucosae and skin are exposed to environmental antigens and are natural entry routes for most infectious agents. To maintain immunological tolerance and ensure protective immunity against pathogens, epithelial surfaces are surveyed permanently by antigen-presenting dendritic cells (DCs).
Many DC subsets have been described in epithelial tissues, depending on the inflammatory state and the type of epithelium. Identification of the DC subset able to induce cytotoxic CD8 ${ }^{+} T$ cells against antigens delivered via mucosae or skin, is a major issue for the development of efficient antiinfectious and anti-tumoral vaccines. Until recently, it was commonly accepted that Langerhans cells (LC), the prototype of immature DCs residing in skin and certain mucosae, can capture and process antigens and, in response to danger signals, undergo a maturation program allowing their migration to the draining lymph nodes for priming of naïve T cells. This concept likely needs to be revisited. Recent evidence from animal models revealed that resident epithelial tissue DCs, including LCs, do not play a direct role in T cell priming, but may contribute to maintenance of peripheral tolerance. Alternatively, DCs newly recruited into mucocutaneous tissues exposed to pro-inflammatory stimuli are responsible for efficient priming and differentiation of CD8 ${ }^{+} T$ cells into cytolytic effectors. These DC originate from blood monocytes and can cross-present protein antigens to $C D 8^{+} T$ cells, which subsequently give rise to specific CTL effectors. Remarkably, components derived from bacteria, virus and chemicals capable to enhance CCL20 production in epithelia, promote CCR6-dependent DC recruitment and behave as adjuvants allowing for cross-primed CD8 ${ }^{+}$CTL. These advances in the dynamic and function of epithelial tissue DC provide a rationale for the screening of novel $\operatorname{CD} 8^{+}$ $T$ cell adjuvants and the design of novel mucosal and skin vaccines. $\diamond$

\section{RÉFÉRENCES}

1. Valladeau J. Les cellules de Langerhans. Med Sci (Paris) 2006 ; 22 : 144-8.

2. Nestle FO, Zheng XG, Thompson CB, et al. Characterization of dermal dendritic cells obtained from normal human skin reveals phenotypic and functionally distinctive subsets. J Immunol 1993; 151 : 6535-45.

3. Larregina AT, Morelli AE, Spencer LA, et al. Dermal-resident CD $14^{+}$cells differentiate into Langerhans cells. Nat Immunol 2001 ; 2 : 1151-8.

4. Pavli P, Woodhams CE, Doe WF, Hume DA. Isolation and characterization of antigen-presenting dendritic cells from the mouse intestinal lamina propria. Immunology $1990 ; 70: 40-7$.

5. Rescigno M, Urbano M, Valzasina B, et al. Dendritic cells express tight junction proteins and penetrate gut epithelial monolayers to sample bacteria. Nat Immunol $2001 ; 2: 361-7$.

6. Valladeau J, Saeland S. Cutaneous dendritic cells. Semin Immunol 2005 ; $17: 273-83$.

7. Kissenpfennig A, Henri S, Dubois B, et al. Dynamics and function of Langerhans cells in vivo dermal dendritic cells colonize lymph node areas distinct from slower migrating Langerhans cells. Immunity 2005; $22: 643-54$.

8. Shortman K, Naik SH. Steady-state and inflammatory dendritic-cell development. Nat Rev Immunol 2007 ; 7 : 19-30.

9. Randolph GJ, Inaba K, Robbiani DF, et al. Differentiation of phagocytic monocytes into lymph node dendritic cells in vivo. Immunity 1999; $11: 753-61$.

10. Merad M, Manz MG, Karsunky H, et al. Langerhans cells renew in the skin throughout life under steady-state conditions. Nat Immunol 2002 ; 3: 1135-41.

11. Ginhoux F, Tacke F, Angeli V, et al. Langerhans cells arise from monocytes in vivo. Nat Immunol $2006 ; 7: 265-73$. 
12. Krasteva M, Kehren J, Horand F, et al. Dual role of dendritic cells in the induction and downregulation of antigen-specific cutaneous inflammation. J Immunol 1998; 160 : 1181-90.

13. Jung $S$, Unutmaz $D$, Wong $P$, et al. In vivo depletion of $C D 11 \mathrm{C}(+)$ dendritic cells abrogates priming of CD8 ${ }^{+} \mathrm{T}$ cells by exogenous cell-associated antigens. Immunity $2002 ; 17: 211-20$.

14. Probst HC, Lagnel J, Kollias G, van den Broek M. Inducible transgenic mice reveal resting dendritic cells as potent inducers of CD8 ${ }^{+} \mathrm{T}$ cell tolerance. Immunity 2003 ; 18 : 713-20.

15. Allan RS, Smith CM, Belz GT, et al. Epidermal viral immunity induced by CD8alpha dendritic cells but not by Langerhans cells. Science $2003 ; 301: 1925-8$.

16. Allan RS, Waithman J, Bedoui S, et al. Migratory dendritic cells transfer antigen to a lymph node-resident dendritic cell population for efficient CTL priming. Immunity 2006 ; 25 : 153-62.

17. Bennett $C L$, van Rijn $\varepsilon$, Jung $S$, et al. Inducible ablation of mouse Langerhans cells diminishes but fails to abrogate contact hypersensitivity. I Cell Biol 2005; 169: 569-76.

18. Kaplan DH, Jenison MC, Saeland $S$, et al. Epidermal langerhans cell-deficient mice develop enhanced contact hypersensitivity. Immunity $2005 ; 23: 611-20$

19. Belz GT, Smith CM, Kleinert L, et al. Distinct migrating and nonmigrating dendritic cell populations are involved in $\mathrm{MHC}$ class I-restricted antigen presentation after lung infection with virus. Proc Natl Acad Sci USA $2004 ; 101: 8670-5$

20. Luci C, Hervouet C, Rousseau D, et al. Dendritic cell-mediated induction of mucosal cytotoxic responses following intravaginal immunization with the nontoxic $B$ subunit of cholera toxin. J Immunol $2006 ; 176: 2749-57$.

21. Itano AA, McSorley SJ, Reinhardt RL, et al. Distinct dendritic cell populations sequentially present antigen to CD4 T cells and stimulate different aspects of cell-mediated immunity. Immunity $2003 ; 19: 47-57$

22. McWilliam AS, Napoli S, Marsh AM, et al. Dendritic cells are recruited into the airway epithelium during the inflammatory response to a broad spectrum of stimuli. J Exp Med 1996 $184: 2429-32$.

23. Anjuere F, George-Chandy A, Audant F, et al. Transcutaneous immunization with cholera toxin $B$ subunit adjuvant suppresses $\lg \varepsilon$ antibody responses via selective induction of Thl immune responses. J Immunol 2003 ; $170: 1586-92$

24. Sierro F, Dubois $B$, Coste $A$, et al. Flagellin stimulation of intestinal epithelial cells triggers CCL20 - mediated migration of dendritic cells. Proc Natl Acad Sci USA 2001 ; 98 : 13722-7.

25. Etchart N, Desmoulins PO, Chemin K, et al. Dendritic cells recruitment and in vivo priming of CD8 $8^{+}$CTL induced by a single topical or transepithelial immunization via the buccal mucosa with measles virus nucleoprotein. J Immunol 2001 ; 167 : 384-91.

26. Le Borgne M, Etchart N, Goubier A, et al. Dendritic cells rapidly recruited into epithelial tissues via $\mathrm{CCR} 6 / \mathrm{CCL} 20$ are responsible for $\operatorname{CD} 8^{+} \mathrm{T}$ cell crosspriming in vivo. Immunity 2006 ; $24: 191-201$
27. Desvignes C, Esteves F, Etchart N, et al. The murine buccal mucosa is an inductive site for priming class I- restricted CD $8^{+}$effector T cells in vivo. Clin Exp Immunol $1998 ; 113: 386-93$.

28. Salazar-Gonzalez RM, Niess JH, Zammit DJ, et al. CCR6-mediated dendritic cell activation of pathogen-specific T cells in Peyer's patches. Immunity 2006; $24: 623-32$.

29. Leon B, Lopez-Bravo M, Ardavin C. Monocyte-derived dendritic cells formed at the infection site control the induction of protective T helper 1 responses against Leishmania. Immunity 2007 ; 26 : 519-31.

30. Belz GT, Behrens GM, Smith CM, et al. The CD8alpha dendritic cell is responsible for inducing peripheral self-tolerance to tissue-associated antigens. J Exp Med 2002 ; 196 : 1099-104.

31. Hawiger D, Inaba K, Dorsett $Y$, et al. Dendritic cells induce peripheral T cell unresponsiveness under steady state conditions in vivo.J Exp Med $2001 ; 194: 769-79$.

32. Akbari 0, DeKruyff RH, Umetsu DT. Pulmonary dendritic cells producing IL-10 mediate tolerance induced by respiratory exposure to antigen. Nat Immunol $2001 ; 2: 725-31$

33. Williamson $\varepsilon$, Bilsborough JM, Viney JL. Regulation of mucosal dendritic cell function by receptor activator of NF-kappa B (RANK)/RANK ligand interactions: impact on tolerance induction. J Immunol 2002 ; $169: 3606-12$.

34. Moingeon P, Batard T, Fadel R, et al. Immune mechanisms of allergenspecific sublingual immunotherapy. Allergy 2006; 61 : 151-65.

35. Geissmann F, Jung S, Littman DR. Blood monocytes consist of two principal subsets with distinct migratory properties. Immunity 2003; 19: 71-82.

36. Vanbervliet B, Homey B, Durand I, et al. Sequential involvement of CCR2 and CCR6 ligands for immature dendritic cell recruitment: possible role at inflamed epithelial surfaces. Eur J Immunol 2002 ; 32 : 231-42.
TIRÉS À PART

D. Kaiserlian

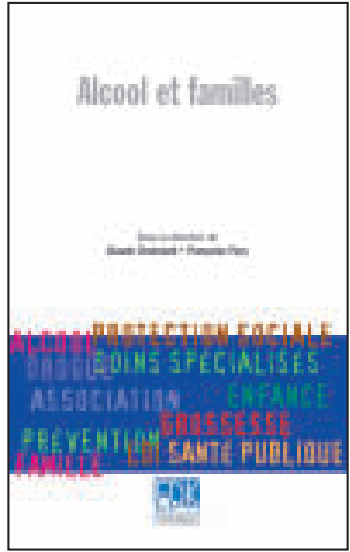

ISBN : 978-2-8425-4116-3 174 pages

\section{Bon de commande}

À retourner à EDK, 2, rue Troyon - 92316 Sèvres Cedex

Tél. : 0155641393 - Fax : 0155641394 - E-mail : edk@edk.fr

NOM :

Prénom :

Adresse :.

Code postal :

Ville :

Pays :

Fonction :

Je souhaite recevoir l'ouvrage Alcool et familles : $15 €+3 €$ de port $=18 €$ TTC

en . exemplaire, soit un total de $€$

$\square$ Par chèque, à l’ordre de $\mathbf{E} \mathbf{D} \mathbf{K}$

$\square$ Par carte bancaire :

$\square$ Visa $\square$ Eurocard/Mastercard

Carte $n^{\circ}$

Date d'expiration :

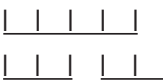

$\mathrm{N}^{\circ}$ de contrôle au dos de la carte :
Signature : 